Int. J. Dev. Biol. 58: 9-19 (2014)

doi: $10.1387 / \mathrm{ijdb} .130351 \mathrm{mb}$

\title{
Mechanisms of cranial placode assembly
}

\author{
MARIE ANNE BREAU* and SYLVIE SCHNEIDER-MAUNOURY \\ Sorbonne Universités, UPMC Univ Paris 06, UMR7622, Paris, CNRS, UMR7622, Paris \\ and INSERM, U1156, Paris, France
}

\begin{abstract}
Cranial placodes are transient ectodermal structures contributing to the paired sensory organs and ganglia of the vertebrate head. Placode progenitors are initially spread and intermixed within a continuous embryonic territory surrounding the anterior neural plate, the so-called panplacodal region, which progressively breaks into distinct and compact placodal structures. The mechanisms driving the formation of these discrete placodes from the initial scattered distribution of their progenitors are poorly understood, and the implication of cell fate changes, local sorting out or massive cell movements is still a matter of debate. Here, we discuss different models that could account for placode assembly and review recent studies unraveling novel cellular and molecular aspects of this key event in the construction of the vertebrate head.
\end{abstract}

KEY WORDS: cranial placode, sensory organ, sorting-out, cell migration, coalescence

\section{Introduction}

Cranial placodes are transient embryonic structures from ectodermal origin that generate crucial parts of the vertebrate head. Anterior-most placodes give rise to the adenohypophysis, the olfactory sensory epithelium and the eye lens. More posterior placodes include the otic placode which generates the entire inner ear and the statoacoustic ganglion, trigeminal and epibranchial placodes that contribute to a set of ganglia relaying sensation from the face and viscera to the brain, a newly identified placode giving rise to the paratympanic organ and additional mechanosensory lateral line placodes in aquatic vertebrates (O'Neill et al., 2012; Schlosser, 2010).

Despite diverse morphologies and functions, placodes and their derivatives arise from progenitors dispersed in a common horseshoe-shaped ectodermal domain called the pan-placodal or pre-placodal region (PPR), surrounding the anterior neural plate by the end of gastrulation (Fig. 1) (Bailey and Streit, 2006; Schlosser, 2010; Streit, 2008). The continuity of this PPR, revealed by fate maps and expression profiles, contrasts with the split pattern of placodal derivatives observed at later stages, in which discrete placode entities occupy specific positions along the anteroposterior axis, next to the neural tube (Fig. 1) (Bailey and Streit, 2006; Schlosser, 2010; Streit, 2008).

How the PPR field gets subdivided into individual placodes is still poorly understood, and the relative contribution of cell fate changes, apoptosis, local sorting out or large scale morphogenetic movements remains an open question. In this review, we summarise our current understanding of the cellular and molecular mechanisms involved in placode assembly, using examples from studies mostly performed with chick, Xenopus, and zebrafish embryos. We focus on the initial formation of discrete and individualised placodal structures. The long-distance migration of the lateral line placodes and the underlying mechanisms have been described elsewhere (Aman and Piotrowski, 2011; Ma and Raible, 2009) and will not be covered here, neither will the complex morphogenetic remodeling of sensory organs occuring at later stages, such as invagination of olfactory and otic placode epithelia.

Based on insights from fate mapping experiments, we propose that the assembly of placodes involves two sequential steps during development, initial segregation of their precursors and secondary coalescence, and we use this view as a framework for discussing the mechanisms underlying these two events.

\section{Fate mapping suggests a two-step placode assembly}

\section{Definition of the pre-placodal region}

The embryonic origin of cranial placodes was initially analysed by fate mapping studies based on transplantation experiments (reviewed in Schlosser, 2010). More recently, dye labelling lineage tracing was performed in fish (Kozlowski et al., 1997; Whitlock and Westerfield, 2000; Dutta et al., 2005), amphibians (Pieper et al., 2011) and birds (Bhattacharyya et al., 2004; Bhattacharyya and Bronner, 2013; Streit, 2002; Xu et al., 2008). To generate these fate

Abbreviations used in this paper: PPR, pre-placodal region.

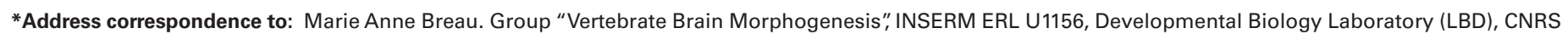
UMR7622, Université Pierre et Marie Curie, 9 Quai Saint-Bernard, Bât C 7ème étage, case 24, 75252 Paris Cedex 05, France.

Tel: +33-1-4427-2153. E-mail: marie.breau@snv.jussieu.fr
} 
A 1s stage

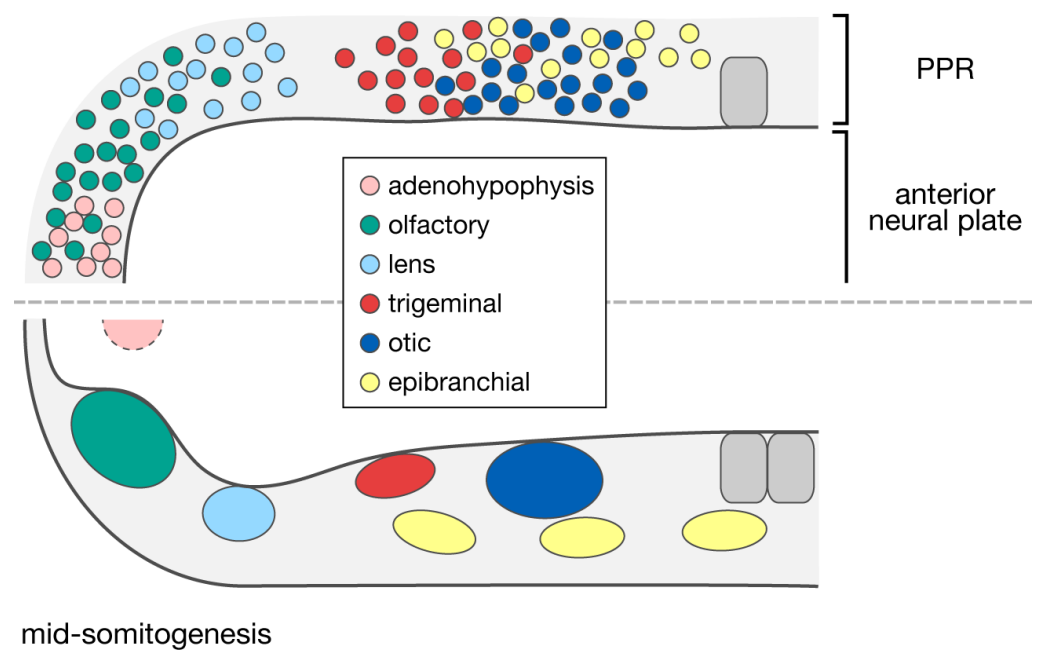

Fig. 1. Position of placodal precursors and placodal structures before and after placode assembly. (A) Schematic view of placode progenitors scattered and intermixed within the pan-placodal region (PPR) surrounding the anterior neural plate at the 1s stage (dorsal view of a theoretical vertebrate). (B) Compact and individualised placodes occupying specific positions along the anteroposterior axis of the embryo from mid-somitogenesis stages onwards (dorsal view, the ventral position of the adenohypophysis is indicated by the dotted line surrounding the placode). Anterior to the left.

maps, dyes were injected or uncaged in single cells or small patches of the head ectoderm at various developmental stages, ranging from gastrulation to late somitogenesis. The fate and location of the progeny was then assessed at later stages, when placodes can be identified by their position and morphology. These studies showed that sensory placodes arise from a crescent-shaped ectodermal territory surrounding the anterior neural plate at late gastrulation/ early neurulation stages. This contiguous region coincides with the expression domain of transcription factors such as Eya1, Six $1 / 2$ and Six $4 / 5$, which are crucial for placode formation and are thought to establish a placodal bias in this domain (Lleras-Forero and Streit, 2012; Pieper et al., 2011; Schlosser, 2010). These observations, together with unique properties shared by cells within this territory (Bailey et al., 2006; Martin and Grooves, 2006), argue that it represents the common domain of origin for all placodes, the so-called pan-placodal or pre-placodal region (PPR) (Bailey and Streit, 2006; Schlosser, 2010; Streit, 2008).

\section{Initial segregation and secondary coalescence}

Although information is still incomplete in some species about subsets of placodes, acommonfeature of these fate mapping studies is the initial overlap between the domains of origin of the different placodes (the extent of this overlap has been recently discussed and challenged, for details see Pieper et al., 2011; Schlosser, 2010), which progressively decreases as development proceeds. That is, the precursors of a given placode appear scattered within the PPR domain and partially intermingled with the precursors of adjacent placodes as well as with other ectodermal cells such as epidermal, neural tube or neural crest cells (NCC), and undergo progressive segregation over time, becoming confined to a particular region (Fig. 1) (Kozlowski et al., 1997; Bhattacharyya et al., 2004; Bhattacharyya and Bronner, 2013; Dutta etal., 2005; Pieper et al., 2011;
Streit, 2002; Whitlock and Westerfield, 2000; Xu et al., 2008). This initial segregation leads to the formation of distinct placodal compartments that are still apposed to each other at early somitogenesis stages (Fig. 2). However, placode assembly is not yet fully completed at this stage. From mid/end of somitogenesis, placodal structures become more compact and clearly separated from each other by non-placodal areas (Figs. 1 and 2) (Schlosser, 2010; Schlosser and Ahrens, 2004; Streit, 2008). This splitpattern and physical separation contrast with the close proximity of placodal domains observed shortly after their initial segregation (Fig. 2).

These observations therefore suggest a two-step sequence in placode formation: initial segregation of placodal precursors into adjacent placodal areas, followed by further compaction to form separated and discrete placodal structures (Fig. 2, the table indicates the developmental stages corresponding to these two steps reported in zebrafish, Xenopus and chick embryos).

The compaction step, referred to as secondary coalescence, is well illustrated by the early morphogenesis of the olfactory placode in zebrafish. Whitlock and Westerfield (2000) performed a fate map of the anterior neural plate at the $4-5$ somites (4-5s) stage, which shows that the paired olfactory placodes arise from two elongated stripe-shaped cell fields flanking the neural plate. Importantly, no lens precursors were found to be intermixed with olfactory placode progenitors at 4-5s, suggesting that the segregation step is already achieved at this stage for these two placodes in zebrafish. Comparison of the shape of the two initial domains at 4-5s with that of the two spherical olfactory placodes seen from 20 s reveals that a coalescence process takes place between these two stages, after segregation with lens progenitors, and before further tissue remodeling by invagination of the epithelium (Whitlock and Westerfield, 2000). This is consistent with the fate mapping of olfactory and lens precursors in chick embryos, which reveals intermingling until 4-5s stage, when the two placodal domains are still juxtaposed to each other, followed by subsequent spatial separation of the two cell populations at later stages (Bhattacharyya et al., 2004; Bhattacharyya and Bronner, 2013).

The driving forces that control initial segregation and secondary coalescence of placodes are still poorly characterised. Both steps could be regulated by similar mechanisms (differential adhesion or chemotactism could for instance mediate cell segregation and further compaction of placodes) or involve distinct cellular processes. Recent studies uncovered novel aspects of the secondary coalescence phase (see part C). However, the initial segregation is still an unsolved issue, and the underlying mechanisms are currently under vigorous debate in the field.

\section{Mechanisms driving the initial segregation of placodal precursors}

How do placodal precursors form segregated embryonic domains from their scattered and intermixed distribution within the continuous PPR? After briefly presenting the two main models that could account for this initial segregation, we discuss old and novel evidence supporting each scenario. 


\section{Two possible scenarios}

Model 1: Large-scale sorting out of early-specified intermingled placodal precursors

According to this model, specification of the progenitors of distinct placodes would precede segregation and provide them with properties allowing them to sort out from each other, leading to the formation of immiscible adjacent pladodal areas. Possible mechanisms to mediate this cell sorting include differential intercellular adhesion, in which randomly moving cells specifically adhere to and aggregate with alike placodal cells (Model 1a, Fig. 3), and active directional migration guided by local sources of secreted or matrix-bound cues that attract or repel distinct placodal cell subpopulations (Model 1b, Fig. 3).

\section{Model 2: Random movements preceding specification of placodal} identities by surrounding signalling centers

In this scenario, multipotent precursors would be exposed to local gradients of environmental signals triggering their specification into particular placodal identities along the anteroposterior axis. Downstream of this signalling, patterning mechanisms such as cross-repression of transcription factors, as seen in the central nervous system, would ensure the formation of a sharp border between placodal domains. The initial intermingling observed in fate maps implies random movements and mixing of the multipotent progenitors within the PPR territory before their specification by extrinsic signals (Model 2, Fig. 3).

\section{Confrontation of the models with experimental evidence}

\section{Are placodal precursors specified before their segregation?}

To sort out from each other, intermingled precursors of future adjacent placodes need to be different, i.e. to have undergone an early specification event that confers them with characteristics allowing their segregation. What is the evidence for such an early specification?

In the fate mapping experiments described above, single labelled cells most often generated progeny in only one specific placode, even when the labelling occured before segregation (Bhattacharyya and Bronner, 2013; Dutta et al., 2005; Xu et al., 2008). This observation suggests that placodal progenitors are lineage-restricted before their spatial segregation (Bhattacharyya and Bronner, 2013; Dutta et al., 2005; Toro and Varga, 2007), although this needs to be more directly proven. The classic assay to estimate the timing of specification for a given cell population consists in dissecting small pieces of tissue at different stages and culturing them in isolation, in order to assess whether cells can differentiate according to their normal fate in a neutral environment. The general outcome of these experiments performed for sub-regions of the PPR is that the onset of placode specification is approximately concomitant with the decrease in the overlap between placodal precursors, which makes it difficult to conclude about the precise temporal sequence linking the two events (Baker et al., 1999; Groves and Broner-Fraser, 2000; Baker and Bronner-Fraser, 2001; Bhattacharyya and Bronner-Fraser, 2008).

Intermingling of early-specified placodal precursors should be reflected by salt-and-pepper expression profiles of placode-specific markers. The formation of segregated placode primordia correlates in time with upregulation of transcription factors specifically expressed in individual or groups of placodes (Bailey and Streit, 2006; Pieper et al., 2011; Schlosser, 2010; Streit, 2008). Pax2 and Pax8, expressed in the otic and epibranchial placode progenitors (and in lateral line placodes in fish and amphibians), show saltand-pepper expression in chick (Groves and Bronner-Fraser, 2000; Streit, 2002), Xenopus (Pieper et al., 2011; Schlosser and Ahrens, 2004), zebrafish (Bhat et al., 2013; Bhat and Riley, 2011; Hans and Westerfield, 2007; McCarroll et al., 2012; Padanad et
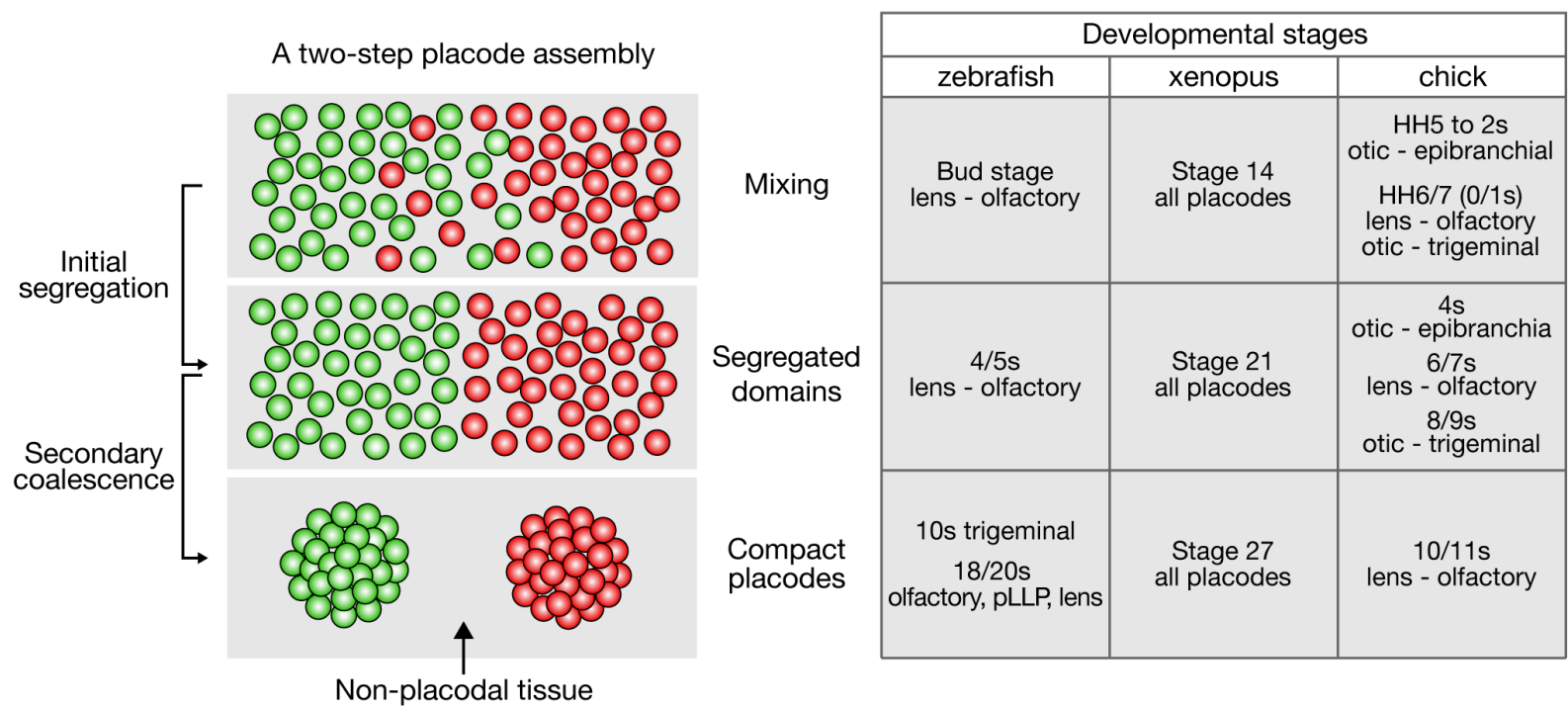

Fig. 2. Placode assembly seen as a two-step developmental process. Fate map studies suggest two sequential steps in placode formation: segregation of intermingled placodal precursors into immiscible but still juxtaposed placodal domains (initial segregation), and further compaction resulting in discrete and condensed placodes separated by non-placodal tissues (secondary coalescence). The table indicates the corresponding developmental stages reported in studies using zebrafish, Xenopus and chick embryos (Data compiled from Kozlowski et al., 1997; Bhattacharyya and Bronner, 2013; Bhattacharyya and Bronner-Fraser, 2004; Breau et al., 2012; Dutta et al., 2005; Harden et al., 2012; Knaut et al., 2005; Kwan et al., 2011; Pieper et al., 2011; Streit, 2002; Whitlock and Westerfield, 2000; Xu et al., 2008). In the upper panel, the colored dots represent progenitors of adjacent placodes, whether or not they are specified. HH: Hamburger Hamilton. pLLP: posterior lateral line placode. s: somite. 
al., 2012) and mice (Ohyama and Groves, 2004). Pax6, a transcription factor expressed in the lens placode, exhibit mosaic expression in zebrafish (Dutta et al., 2005; Hans and Westerfield, 2007). It will be important to understand the mechanisms able to generate these mosaic patterns, such as stochastic activation of gene expression, threshold effects or lateral inhibition within the PPR. However, salt-and-pepper expression could also reflect the progressive up or down-regulation of a placode-specific marker within a contiguous placodal domain, and does not constitute a direct proof of the early specification and intermingling of placodal precursors. Double in situ hybridisations for markers of adjacent placodes would represent stronger evidence, but are almost absent from the literature, except for a double in situ hybridisation performed on zebrafish embryos at bud stage, which suggests intermingling between Pitx3-positive adenohypophysis precursors and DIx3-expressing olfactory precursors (Dutta et al., 2005). Thus, existing data do not resolve the question of when placodal fates are specified.

Do placodal precursors undergo directional movements during segregation?

If the initial segregation step is driven by directional cell movements, these should be detected by live imaging. The first attempts to monitor the dynamic behaviour of placodal progenitors during segregation were made in chick embryos labelled with dye crystal spots in the otic/epibranchial (Streit, 2002) and lens/ olfactory (Bhattacharyya et al., 2004) presumptive regions. In both reports, the movies start at stages when placodal precursors are still intermingled, according to fate maps performed in the same studies (Bhattacharyya and Bronner-Fraser, 2004; Streit, 2002). In the first hours of the time-lapse sequence shown in Streit, 2002, coordinated convergence movements directed towards the midline and affecting the whole embryo can be observed, during which dye-labelled cells remain together. Strikingly, the labelled cells start to spread and migrate away from their initial cluster only when the first $6 / 7$ somites have formed, i.e. when initial segregation of the precursors is already achieved (Fig. 2). Similar observations can be made for the second report: overall convergence towards the midline is followed by splitting of presumptive olfactory and lens cells from stage $5 \mathrm{~s}$, which corresponds to the timing of the secondary coalescence phase, according to fate maps performed in the same study (Bhattacharyya et al., 2004). Thus, in both studies, the documented cell movements likely represent the secondary coalescence process which occurs at later stages, rather than directional migration driving the early segregation of placodal populations.

A recent live imaging study of placodal cell behaviour during segregation stages was performed in Xenopus (Pieper etal., 2011). The authors performed a detailed analysis of cell movements and neighbour exchange, and found no evidence supporting the notion of directional movements. This work argues against extensive long-distance directed cell migration, but does not excude the possibility of local directional rearrangements that would sharpen the boundaries between adjacent placodal domains.

Is the initial segregation driven by adhesion-mediated sorting-out?

Live imaging analysis does not support the notion of largescale directional movements mediating the initial segregation of placodal precursors. One explanation could be that it rather relies on differential adhesion-mediated sorting-out, which can occur without directional movements (Model 1a, Fig. 3). This is supported by functional studies of Pax6 and DIx5 transcription factors, respectively markers for lens and olfactory placode precursors.

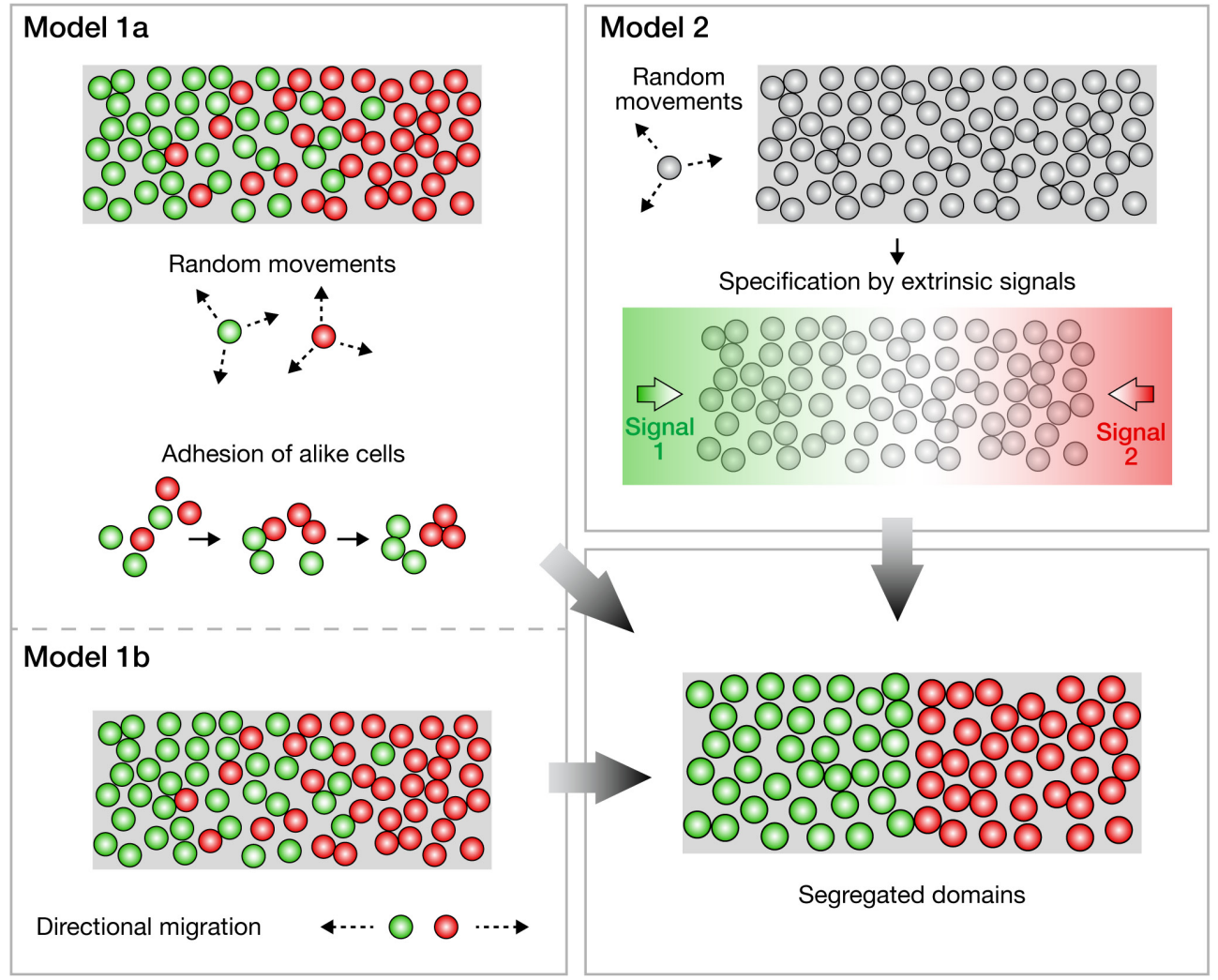

Fig. 3. Two scenarios for the initial segregation of placodal precursors. (Model 1). Large-scale sorting out of early-specified intermingled placodal precursors. Specified placodal precursors initially present a high degree of intermixing and actively sort-out from each other by differential adhesion (Model 1a) or directional migration (Model 1b). (Model2). Random movements preceding specification of placodal identities by surrounding signalling centers. Unspecified progenitors undergo random movements within the PPR before being specified by environmental signals. Further patterning mechanisms (cross-repression of transcription factors) lead to the formation of a sharp boundary between placodal domains. Grey and colored dots represent unspecified and specified placodal progenitors, respectively. 
In mouse chimaeras containing a mixture of $\mathrm{Pax}^{-{ }^{--}}$and wild type cells, Pax6 mutant cells are excluded from the lens (Collinson et al., 2000). This suggests a capacity of Pax6-positive and Pax6negative populations to segregate from each other, although loss of $\mathrm{Pax}^{-1-}$ cells by apoptosis in the lens tissue has not been ruled out. It has been hypothesised that Pax6 regulates the expression of intercellular adhesion molecules responsible for this sorting out process (Collinson et al., 2000), but a more recent study in mouse rather suggests that Pax 6 controls lens formation by regulating the expression of extracellular matrix (ECM) components, including fribronectin (Huang et al., 2011). In zebrafish, forced expression of Dlx5, normally present in a domain anterior and adjacent to the $\mathrm{Pax}^{+}$region (the presumptive olfactory placode domain), leads to the formation of clusters of DIx5-overexpressing cells and their exclusion from the lens placode. Despite the lack of loss-of-function data, this suggests that DIx 5 could control cell sorting by regulating adhesive properties of placodal cells (Bhattacharyya et al., 2004).

\section{Towards a reconciliation of the two models}

The real situation could correspond to an hybrid scenario that reconciles Models 1 and 2, in which extrinsic signals specify adjacent placodal territories with fuzzy boundaries that are further refined and sharpened by local, small-scale active rearrangements, as recently described for the dorso-ventral patterning in the zebrafish spinal cord (Xiong et al., 2013). In this situation, the extent of intermixing is limited, consistent with the idea that this overlap was overestimated due to artefacts in fate mapping experiments (Pieper et al., 2011; Schlosser, 2010).

The hybrid model is supported by the investigation of the local segregation between otic and epibranchial precursors carried out in zebrafish (McCarroll et al., 2012). The authors use Kaede fatemapping to show that otic and a subset of epibranchial progenitors initially occupy adjacent regions within a Pax2 expression domain lateral to the hindbrain, the so-called PPA(Posterior Placodal Area). The main source of epibranchial precursors resides outside of the PPA, in the adjacent, more lateral non-neural ectoderm (Bhat and Riley, 2011; Padanad and Riley, 2011). The authors observe heterogeneous Pax2 expression within the PPA, and ask whether differences in Pax2 expression levels could drive or influence the formation of otic versus epibranchial placodes. Cells overexpressing Pax2 preferentially incorporated into the otic placode and contributed less to epibranchial placodes as compared with control cells. Moreover, they were seen moving towards the otic field in live imaging experiments. Loss-of-function was more complex to achieve due to the requirement of Pax2 for normal epibranchial placode development (Padanad and Riley, 2011), and functional redundancy between Pax2 and Pax8 in the PPA domain. Nevertheless, partial double knockdown of Pax2 and Pax8 led to an increased number of epibranchial placodal cells, combined with a reduction (although not statistically significant) of otic vesicle size. Together, these results suggest that otic and the subset of epibranchial precursors found in the PPA locally segregate from each other according to Pax2/8 expression levels, with high and low expressing cells preferentially contributing to the otic and epibranchial placodes, respectively. The authors further show that Wnt signalling induces high expression of Pax2 within the PPA and promotes otic placode formation. They propose a model in which specification by an extrinsic signal (Wnt secreted by the neural tube) leads to the presence of two adjacent cell populations (otic and epibranchial precursors) separated by a fuzzy border, which locally segregate from each other to contribute to distinct placodes (McCarroll et al., 2012). This model will need to be confirmed by an non-equivocal visualisation of the local intermixing between the two cell populations within the PPA, and the detailed characterisation of their behaviour and fate during the segregation of otic and epibranchial placodes.

Thus, despite important progress, the issue of initial segregation still deserves further investigation. To tackle this fundamental question, the ability to follow single cell fates over and after segregation, and to correlate cell movements with potential changes in gene expression, will be decisive. This could be achieved with transgenic lines specifically labelling adjacent populations of placodal precursors with different colors. The construction of such tools is now feasible by knocking-in with engineered nucleases techniques (Auer et al., 2013). A potential difficulty is that placode segregation occurs during overall convergence movements towards the midline of the embryo (Bhattacharyya and Bronner-Fraser, 2004; Streit, 2002). It will be challenging to capture potential short-distance directional cell displacements within these converging fields of cells, but this can be done by a careful analysis of neighbour exchange.

\section{Morphogenetic movements involved in secondary placode coalescence}

Once placodal cells occupy defined areas with no or poor intermingling, how is the physical separation of placodes achieved?

Many mechanisms could account for placode condensation and physical separation, including cell fate transitions, cell death, changes in cell shapes, and passive or active cell movements. To our knowledge, no experimental data support the contribution of cell fate changes in the secondary coalescence phase. Apoptotic cells were observed at interplacodal boundaries during the separation between otic and epibranchial areas, and later during the resolution of the epibranchial placode into discrete clusters in mammals (Knabe et al., 2009; Washausen and Knabe, 2012; Washausen et al., 2005), but their functional importance in placode separation has not been assessed. Cell shape changes such as a transition from flattened to epithelial morphology (mesenchyme to epithelium transition), or a reduction in placodal cell volume, could both result in overall compaction of placodal areas, but such morphological transitions have not been described so far. Placodal cells could be passively pushed or tracted to their final location by large-scale movements, folding or growth of surrounding tissues. For example, the outgrowth of the retina may contribute to the separation of olfactory and lens progenitors, although this needs to be experimentally tested.

Here, we focus on recent reports that point to a significant contribution of active morphogenetic movements to the secondary placode coalescence, and summarize our current knowledge of the cell behaviours and mechanisms involved.

\section{Diverse behaviours and movements \\ Delamination}

Trigeminal and epibranchial (geniculate, petrosal and nodose) placodes are neurogenic patches embedded in the surface ectoderm, which produce neurons that meet and coalesce with NCC to form the cranial ganglia in the underlying mesenchyme (D'Amico-Martel and Noden, 1983). The ganglia thus condense 
at a distance from the placodal ectoderm, suggesting the implication of detachment from the ectoderm (delamination) and inward migration of placodal cells. We consider these processes as taking part in the secondary coalescence of placodes, as they start shortly after the end of placodal precursor segregation (Xu et al., 2008; Stark et al., 1997), and contribute to generate discrete and condensed placodal-derived ganglia by the end of somitogenesis. In the otic placode, another neurogenic placode, neuroblasts also detach from the epithelium to form the statoacoutstic ganglion, but this delamination step affects only a fraction of otic placodal cells (Schneider-Maunoury and Pujades, 2007).

The delamination process of trigeminal and epibranchial placodes was initially observed on sections of fixed tissues in mammals and in chick embryos (for example, see Blentic et al., 2011; Graham et al., 2007; Knabe et al., 2009; McCabe et al., 2009; Shiau et al., 2008; Xu et al., 2008), and by Dil labelling of the surface ectoderm in chick (Stark et al., 1997; Begbie, 2001a; McCabe et al., 2009). However, delamination of trigeminal or epibranchial cells from the ectoderm has to our knowledge not yet been directly observed in zebrafish or amphibian embryos, and its timing and modalities remain unclear in these species. Lately, a transverse slice culture system was developed to follow dynamic cell behaviours during delamination of trigeminal neuroblasts in chick (Shiau et al., 2011). The movies show neurons detaching from the surface ectoderm as individual cells or as chains of cells connected by axon-like protrusions. The delamination of trigeminal/epibranchial cells, which occurs after the initiation of neurogenesis in the placode areas (Begbie, 2002; McCabe et al., 2009), appears to be distinct from the canonical epithelium-to-mesenchyme transition (EMT) undergone by NCC emerging from the neural tube, since placodal cells seem to leave the ectoderm as neurons and, at least in the epibranchial placode, do not express the Snail transcription factor known to control EMT (Fig. 4) (Graham et al., 2007).

\section{Directional cell movements}

As we mentioned earlier, the extensive movements observed in live imaging studies performed in chick embryos most likely correspond to the coalescence phase (Bhattacharyya and BronnerFraser, 2004; Streit, 2002). However, in the absence of single cell resolution, it is difficult to conclude whether the movements of the dye spots reflect true directional cell migration or passive movements following the morphogenesis of surrounding tissues.

Single cell resolution of lens cell behaviour has been obtained in a dynamic analysis of optic cup morphogenesis in zebrafish (Kwan et al., 2011), in which the eye region was imaged fom 6s onwards, after lens/olfactory segregation. 3D manual cell tracking shows that lens precursors converge towards the top of the retina by directed antero-posterior and postero-anterior movements, before undergoing shape changes resulting in the invagination of the lens placode. Interestingly, antero-posterior migrating lens cells move in coordination with underlying retinal cells, suggesting a possible interaction between the two cell populations (Kwan et al., 2011). Two other recent reports uncovered convergence movements during olfactory and otic placode coalescence in zebrafish. Overall antero-posterior convergence movements of placode precursors accompany olfactory placode coalescence between $4 \mathrm{~s}$ and $18 \mathrm{~s}$ stages (Harden et al., 2012), whereas combined convergence movements along three directions (latero-medial, antero-posterior and postero-anterior) lead to the formation of the otic placode next to the hindbrain between 4s and 11s (Bhat and Riley, 2011).

As described above, the shapes and behaviour of trigeminal and epibranchial cells have been nicely captured in chick embryos during and shortly after delamination of cells into the mesenchyme, but their behaviour during migration and ganglia aggregation has not been documented. Alikely explanation is that ganglion coalescence requires movements along the antero-posterior axis that cannot be visualised on transverse sections or with a slice culture assay. Indeed, live imaging on dorsal views of whole-mount zebrafish embryos uncovered a striking antero-posterior component in the migration of trigeminal placodal cells before their condensation into ganglia. In zebrafish, trigeminal neuron precursors appear initially scattered within two elongated domains along the brain (Bhat and Riley, 2011; Knaut et al., 2005). Time-lapse imaging revealed that between 4 s and 10 s stages, anterior trigeminal neuronal progenitors migrate directionally as isolated cells or with a chain-like pattern towards less motile posterior cells and fuse with them to form a compact cluster next to the midbrain/hindbrain boundary (Fig. 4) (Bhat and Riley, 2011; Knaut et al., 2005).

Altogether, these live imaging studies clearly show that the secondary coalescence of several cranial placodes implicates directional cell movements. The link between cell migration and delamination remains however elusive in several cases. Whereas the migration of trigeminal/epibranchial cells towards the site of ganglion aggregation clearly follows delamination in mammals and chick, it remains unclear whether placodal cells migrate freely in the mesenchyme underlying the ectoderm, or crawl on or even move within the ectodermal layer in all the other situations described above. Moreover, in mammals and chick, whether trigeminal/epibranchial placodal cells undergo coalescence movements within the surface ectoderm before the delamination process needs to be clarified. This will require a careful examination of the position of placodal cells in relation to the ectoderm during their coalescence movements.

\section{Cell retention}

Another cellular process involved in placode coalescence is cell retention, in which placodal cells are trapped at the site of placode formation and prevented from moving away by active mechanisms such as chemotactism or cell/matrix adhesion.

This scenario has for instance been described for the coalescence of the posterior lateral line placode in zebrafish. This placode, found in aquatic vertebrates, generates a group of cells (the primordium) which migrates from head to tail along the somitic myoseptum to deposit superficial sensory organs called neuromasts (Aman and Piotrowski, 2011; Ma and Raible, 2009). We observed (Breau et al., 2012) that prior to the onset of this antero-posterior migration, the compact state of the primordium is not established, as isolated cells with lateral line placode identity are present ahead of the primordium. Although these isolated cells appear static, we showed that they are actively maintained in position by a chemotactic mechanism, in the absence of which they migrate away from the primordium (Fig. 4). This cell retention facilitates the fusion of the isolated placodal cells with the primordium as it advances (Breau et al., 2012). In a similar way, trigeminal neuroblasts also appear to be actively retained by chemotactism at the site of ganglion aggregation in zebrafish (Knaut et al., 2005).

Studies in chick and mouse revealed that lens placode formation is correlated with an increase in cell density in the ectoderm 
overlying the retina, while the contact area between the retina and the surrounding ectoderm does not change. During this process, lens cells change their shape from cuboidal to columnar, but do not proliferate more or become smaller in volume than pre-placodal ectodermal cells (Huang et al., 2011). Huang and co-workers attributed this local increase in cell density to continued proliferation of lens cells combined with their anchoring to the underlying ECM, a retention mechanism that would work against the expansion of the placodal ectoderm. However, cell crowding at the site of lens placode assembly could also result from incoming migrating cells, as reported in zebrafish (Kwan et al., 2011).

Thus, placode coalescence can involve various types of movements and behaviours, including delamination, directional individual and chain migration, collective convergence or active cell retention. What are the underlying molecular mechanisms?

\section{Cellular interactions and molecular cues}

Interaction between placodal and neural crest cells

An accumulating body of evidence shows that interactions between placodal and cranial NCC guide trigeminal/epibranchial placodal cells during their delamination, inward migration and coalescence. Close proximity between the two cell types has been shown in a number of studies at several stages of gangliogenesis (for instance, see Begbie, 2001; Shiau et al., 2008; Shigetani et al., 2008; Theveneau et al., 2013), the most recent one reporting the intriguing observation that NCC form corridors that wrap around migrating epibranchial neuroblasts in chick and mouse (Freter et al., 2013). Contradictory results were obtained from cell ablation experiments attempting to unravel a functional interaction between the two cell populations. Whereas NCC depletion had no consequence on the development of the facial (geniculate) ganglion in mice (Coppola et al., 2010), NCC ablation in chick perturbed the coalescence of cranial ganglia without affecting delamination of placodal cells (Shiau et al., 2008), or led to early delamination defects in another study (Begbie, 2001). A recent report demonstrated with genetic ablation of subtypes of NCC that chondrogenic NCC, but not gliogenic NCC, are required for initial epibranchial ganglia formation in zebrafish (Culbertson et al., 2011). This suggests that the conflicting results obtained in various contexts of NCC depletion might come from differences in the nature of NCC subpopulations affected by each ablation procedure (Culbertson et al., 2011).

In chick, the Slit/Robo system mediates at least partly the interaction between NCC and trigeminal placodal cells. NCC express the Slit1 ligand, whereas placodal cells express its cognate receptor Robo2, and functional perturbation of this ligand-receptor pair leads to disorganised trigeminal ganglia (Shiau et al., 2008). The same authors further showed that N-Cadherin acts in cooperation with Slit1/Robo2 signalling to mediate coalescence of the trigeminal ganglion (Shiau and Bronner-Fraser, 2009).

The coalescence of discrete epibranchial placodes from the initial epibranchial placodal domain has recently been shown to involve an original mechanism of reciprocal interactions between NCC and placodal cells in Xenopus (Theveneau et al., 2013). This study clearly demonstrates that epibranchial placodal cells migrate
A

\section{Delamination}

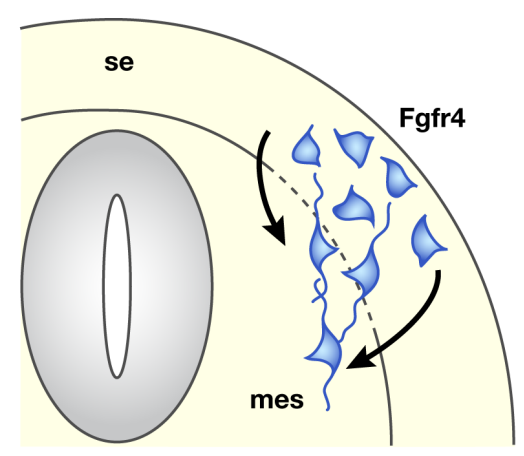

Trigeminal/epibranchial placodes (chick)

C
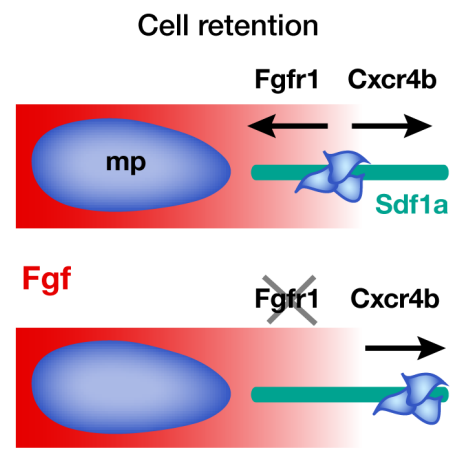

Posterior lateral line placode (zebrafish)
B

Directional cell movements

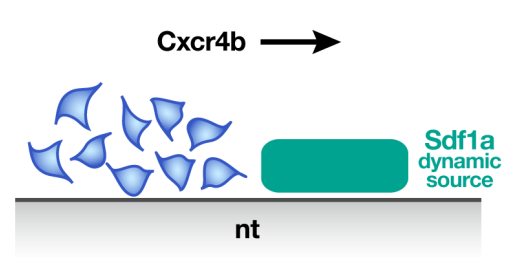

Fig. 4. Examples illustrating active morphogenetic mechanisms that drive secondary placode coalescence. (A) Delamination. Trigeminal and epibranchial placodal cells delaminate from the surface ectoderm (se) as neurons before migrating in the underlying mesenchyme (mes) and coalescing into sensory ganglia. In chick, this process depends on the function of the Fgfr4 receptor expressed by placodal cells (Lassiter et al., 2009). Dorsal to the top. nt: neural tube. (B) Directional cell movements. In zebrafish, Cxcr4b-expressing trigeminal neurons undergo an antero-posterior migration before condensing into ganglia. They move as isolated cells or with a chain-like pattern towards a dynamic source of Sdf1a cue (Knaut et al., 2005; Lewellis et al., 2013). Anterior to the left. nt: neural tube. (C) Cell retention. In zebrafish, before the migration onset of the posterior lateral line primordium, the compact state of the primordium is not fully established, as isolated cells with lateral line placode identity are present ahead of the main primordium (mp). The static position of these cells results from two opposing chemotactic signals. Cxcr4b/Sdf1a signalling attracts the cells away from the primordium, whereas Fgf ligands secreted by the main primordium attract them in the opposite direction, thereby retaining them in the vicinity of the placode. Thus, upon inhibition of Fgf signalling, the cells move away in a Cxcr4b/Sdf1a-dependent manner. This chemotactic cell retention mechanism facilitates the coalescence of the isolated cells with the main primordium into a compact group of migrating cells (Breau et al., 2012). Anterior to the left. 
ventrally and separate into distinct streams once they are contacted by the adjacent and more dorsal cranial NCC. This results from a chase-and-run behaviour, in which NCC are attracted by placodal cells, and placodal cells are in turn repelled by NCC (Theveneau et al., 2013).

\section{Cell/matrix adhesion}

Morphogenetic movements most often rely on interactions between cells and ECM components, which are mainly mediated by integrin receptors. Bhat and Riley performed a functional analysis in zebrafish of itga5, a gene encoding an alpha integrin subunit expressed in the PPR domain from 1s stage and in the placodes during coalescence (Bhat and Riley, 2011). Morpholino-mediated knockdown leads to disorganised posterior placodes (trigeminal, epibranchial and otic placodes), and live imaging shows that cell behaviours are more erratic during convergence movements accompanying otic placode coalescence and the antero-posterior migration of trigeminal neuroblasts (Bhat and Riley, 2011). Itga5containing integrins are thus required for proper coalescence of posterior placodes, and it will be interesting to identify the ligands that mediate this effect. Moreover, anterior placodes are not affected in itga5-deficient embryos, suggesting that anterior placode assembly relies on other integrin receptors or other adhesion systems to be identified.

In mouse embryos, the increase in cell density observed during lens formation correlates with the accumulation of fibronectin, a major ECM component, between the retina and the overlying placodal ectoderm (Huang et al., 2011). Knocking-out the fibronectin gene disrupts cell crowding in the lens placode, which appears larger than in controls (Huang et al., 2011). This result suggests that fibronectin works as a retention/anchoring cue for lens cells located above the retina (as proposed by the authors). Alternatively, fibronectin could act as a cue guiding the directional migration of lens precursors towards the site of placode formation.

\section{Chemokine signalling}

The Cxcr4b/Sdf1a(Cxcl12a) chemokine pathway, which guides the movements of many cell types during development, adult life and in pathologic conditions such as cancer, controls the assembly of olfactory, trigeminal and epibranchial placodes.

In zebrafish, the $\mathrm{Cxcr} 4 \mathrm{~b}$ receptor is expressed in olfactory placode progenitors surrounding the Sdf1a-producing telencephalon. In cxcr4b mutants, a subset of olfactory placode cells occuppy aberrant anterior positions by the end of the coalescence process (Miyasaka et al., 2007). It has been suggested that Cxcr4b/Sdf1a works as a retention signal against the overall anterior morphogenetic stream that takes place in the head region at these stages (Miyasaka et al., 2007). However, live imaging recently revealed that olfactory placode cells, instead of being retained in position, move towards the posterior zone of the olfactory field to form the two compact placodes (Harden et al., 2012), suggesting that their anterior mis-positionning in $c x c r 4 b$ mutants could result from their impaired antero-posterior movements. This will have to be confirmed by time lapse imaging. Moreover, as placode assembly is only partially affected in cxcr4b mutants (Miyasaka et al., 2007), other molecular mechanisms must be at work and remain to be identified.

Cxcr4b/Sdf1a chemokine signalling also guides the anteroposterior migration and coalescence of trigeminal neurons in zebrafish. Cxcr4b is expressed by trigeminal neurons, whereas the
Sdf1a cue is produced by surrounding cells of unknown identity, posterior to the sensory neurons, near the site of ganglion assembly (Fig. 4). In Cxcr4b/Sdf1a-deficient embryos, anterior neurons fail to properly migrate towards and coalesce with more posterior cells, and neurons are seen escaping from small clusters, suggesting that the Cxcr4b/Sdf1a pathway not only guides antero-posterior migration of trigeminal neurons, but also maintains them in the ganglion cluster, acting as a retention signal. Importantly, this attraction and retention mechanisms seem to work against anterior morphogenetic movements of the surrounding tissues, as shown by labelling of cells in the environment of the placodal cells (Knaut et al., 2005). A second study further showed that another receptor for Sdf1a, Cxcr7b, is required for the migration of trigeminal neurons (Lewellis et al., 2013). Consitent with its expression in the close environment of the migrating neurons, Cxcr $7 b$ is required in the vicinity of the neurons, rather in the neurons themselves (Lewellis et al., 2013), unlike Cxcr4b which acts cell-autonomously (Knaut et al., 2005). Double Cxcr4b/Cxcr7b mutants show migration defects that are not more drastic than in single mutants, suggesting that the two receptors work in the same pathway. Overexpression of Cxcr7b in the vicinity of the neurons disturbs their mirgation. Moreover, using internalisation of a Cxcr4b-GFP fusion protein as a readout for active Sdf1/Cxcr4b signalling, the authors demonstrate that $\mathrm{Cxcr} 7 \mathrm{~b}$ decreases this signalling. These results suggest that Cxcr7b inactivates Sdf1 by acting as a sink for this chemokine, as shown in other systems (Boldajipour et al., 2008; Donà et al., 2013; Venkiteswaran et al., 2013). The authors propose a model in which Cxcr7b works in synergy with a Sdf1 mRNA clearance system mediated by a microRNA, miR-430; together they dynamically sculpt the source of Sdf1 in such a way that it is always closely associated with and ahead of migrating trigeminal neurons, therefore guiding them along the antero-posterior axis (Fig. 4, Lewellis et al., 2013). Thus, if Cxcr7b and Cxcr4b likely compete for Sdf1a ligand binding at the molecular level, they act together to promote the migration of trigeminal neurons. The tight spatiotemporal control of the chemokine source would prevent trigeminal neurons from responding to other nearby sources of Sdf1a attractant such as the telencephalon in between the two olfactory placodes (Lewellis et al., 2013).

Finally, Cxcr4b/Sdf1a chemokine signalling mediates the chase behaviour in the reciprocal interactions of NCC and epibranchial placodes described by Theveneau and colleagues (Theveneau et al., 2013). In this situation, placodal cells play the role of the Sfd1a source that attracts Cxcr4b-expressing NCC. In turn, contacts between NCC and placodes repel placodal cells, a process that requires Wnt-PCP and $\mathrm{N}$-cadherin signalling. This mechanism ensures a constant displacement of the Sdf1a source and coordinated movements of the two adjacent cell populations, which results in the separation of the epibranchial placodal domain into distinct digit-shaped streams of cells (Theveneau et al., 2013).

\section{Fgf signalling}

Beside its known role in placode induction, specification and differentiation (Bailey et al., 2006; Freter et al., 2008; Ladher, 2005; Maier et al., 2010; Martin, 2006; Nechiporuk et al., 2006; Nikaido et al., 2007; Sun et al., 2007), Fgf signalling controls some aspects of the movements and behaviours driving placode coalescence.

The Fgfr4 receptor is transiently expressed in cells of the trigeminal placode during the delamination process in chick em- 
bryos. Expression of dominant negative forms of Fgfr4 prevents the delamination of placodal cells, which eventually loose their trigeminal identity and their capacity to differentiate into sensory neurons (Fig. 4) (Lassiter et al., 2009). The nature and localisation of the Fgf cue controlling this process remain to be identified.

We mentioned earlier that in zebrafish, isolated placodal cells are located ahead of the posterior lateral line primordium before its migration onset. We showed that upon inhibition of Fgf signalling, these isolated cells move away from the primordium, demonstrating that Fgf activity is required for their maintenance in the vicinity of the placode. We further showed that this retention is mediated by Fgf chemotactic ligands secreted by the primordium (Fig. 4) (Breau et al., 2012). This mechanism facilitates the coalescence of the placode into a compact group of migrating cells. The forward migration of the isolated cells observed in the absence of Fgf signalling is due to Cxcr4b/Sdf1a-mediated attraction, which later controls the antero-posterior migration of the primordium (Fig. 4) (Aman and Piotrowski, 2011; Ma and Raible, 2009). Thus, while Cxcr4b/ Sdf1a signalling is necessary for the coalescence of several placodes, it works against the initial compaction of the posterior lateral line placode, which requires the counteracting effect of Fgf cues.

\section{Conclusions and future directions}

In conclusion, we propose to see placode assembly as a sequence of two events, the early segregation of placodal precursors and their subsequent coalescence into discrete placodal derivatives. This view provides a comprehensive framework that facilitates our interpretation and understanding of old and novel experimental data obtained in the field of cranial placode development.

A better knowledge of the mechanisms underpinning both steps of placode assembly will require to revisit fate map studies with state-of-the-art lineage tracing tools, such as those available in the zebrafish model (Cre/Lox technology, local protein photoactivation), combined with high-resolution live imaging of behaviour, polarity/ shape changes, and intracellular dynamics of placodal cells.

There is increasing evidence supporting the importance of cell movements and active retention during the secondary placode coalescence phase. Cxcr4b/Sdf1a chemokine signalling appears to be commonly used by different placodal cell types during coalescence. It will be interesting to determine whether the Fgf pathway, mostly known for its implication in early placode induction/specification or late remodelling of placodal-derived tissues (Lecaudey et al., 2008; Sai and Ladher, 2008), also plays a common role in the morphogenetic mechanisms driving placode assembly. A major challenge will be to identify the intracellular regulators that translate these signals into cell shape changes and migratory behaviours, as the diversity of placode morphologies may come from differences in their way of interpreting and transducing these common cues. The discovery of new molecular players will benefit from techniques such as RNA sequencing, chemical and genetic screens, and emerging genome editing approaches using engineered endonucleases.

Finally, during neurogenic placode formation, placodal-derived neurons not only have to find their proper location, but also face the challenge of establishing appropriate connections with their peripheral and central target tissues to form functional neuronal circuits relaying sensory information to the brain. How the assembly of placodal-derived structures is coordinated with the initiation of this innervation represents another fascinating, yet unexplored ques- tion in the developmental biology of sensory organs and ganglia.

\section{Acknowledgements}

We would like to thank Martin Catala, François Giudicelli, Guillaume Pézeron and Christine Vesque for critical reading of the manuscript and helpful suggestions, and Sophie Gournet for artwork. MAB was supported by a fellowship from the Fondation pour la Recherche Biomédicale.

\section{References}

AMAN, A., and PIOTROWSKI, T. (2011). Cell-cell signaling interactions coordinate multiple cell behaviors that drive morphogenesis of the lateral line. Cell Adh Migr 5: 499-508.

AUER, T.O., DUROURE, K., DE CIAN, A., CONCORDET, J.P., and DEL BENE F. (2013). Highly efficient CRISPR/Cas9-mediated knock-in in zebrafish by homologyindependent DNA repair. Genome Res 24: 142-153.

BAILEY, A.P., BHATTACHARYYA, S., BRONNER-FRASER, M., and STREIT, A (2006). Lens Specification Is the Ground State of All Sensory Placodes, from which FGF Promotes Olfactory Identity. Dev Cell 11: 505-517.

BAILEY, A.P., and STREIT, A. (2006). Sensory Organs: Making and Breaking the Pre-Placodal Region. Curr Top Dev Biol 72: 167-204.

BAKER, C.V. and BRONNER-FRASER, M. (2001). Vertebrate Cranial Placodes I. Embryonic Induction. Dev Biol 232: 1-61.

BAKER, C.V., STARK, M.R., MARCELLE, C., and BRONNER-FRASER, M. (1999). Competence, specification and induction of Pax-3 in the trigeminal placode. Development 126: 147-156.

BEGBIE, J., and GRAHAM, A. (2001). Integration Between the Epibranchial Placodes and the Hindbrain. Science 294: 595-598.

BEGBIE, J., BALLIVET, M., and GRAHAM, A. (2002). Early Steps in the Production of Sensory Neurons by the Neurogenic Placodes. Mol Cell Neurosci21: 502-511.

BHAT, N., KWON, H.J. and RILEY, B.B. (2013). A gene network that coordinates preplacodal competence and neural crest specification in zebrafish. Dev Biol 373: 107-117.

BHAT, N., and RILEY, B.B. (2011). Integrin- $\alpha 5$ Coordinates Assembly of Posterior Cranial Placodes in Zebrafish and Enhances Fgf-Dependent Regulation of Otic/ Epibranchial Cells. PLoS One 6, e27778.

BHATTACHARYYA, S., BAILEY, A.P., BRONNER-FRASER, M., and STREIT, A. (2004). Segregation of lens and olfactory precursors from a common territory: cell sorting and reciprocity of DIx5 and Pax6 expression. Dev Biol 271: 403-414.

BHATTACHARYYA, S., and BRONNER, M.E. (2013). Clonal analyses in the anterior pre-placodal region: implications for the early lineage bias of placodal progenitors. Int J Dev Biol 57: 753-757.

BHATTACHARYYA, S., and BRONNER-FRASER, M. (2004). Hierarchy of regulatory events in sensory placode development. Curr Opin Genet Dev 14: 520-526.

BHATTACHARYYA, S., and BRONNER-FRASER, M. (2008). Competence, specification and commitment to an olfactory placode fate. Development 135: 4165-4177.

BLENTIC, A., CHAMBERS, D., SKINNER, A., BEGBIE, J., and GRAHAM, A. (2011) The formation of the cranial ganglia by placodally-derived sensory neuronal precursors. Mol Cell Neurosci 46: 452-459.

BOLDAJIPOUR, B., MAHABALESHWAR, H., KARDASH, E., REICHMAN-FRIED, M., BLASER, H., MININA, S., WILSON, D., XU, Q., and RAZ, E. (2008). Control of Chemokine-Guided Cell Migration by Ligand Sequestration. Cell 132: 463-473.

BREAU, M.A., WILSON, D., WILKINSON, D.G., and XU, Q. (2012). Chemokine and Fgf signalling act as opposing guidance cues in formation of the lateral line primordium. Development 139: 2246-2253.

COPPOlA, E., RALLU, M., RICHARD, J., DUFOUR, S., RIETHMACHER, D., GUILLEMOT, F., GORIDIS, C., and BRUNET, J.F. (2010). Epibranchial ganglia orchestrate the development of the cranial neurogenic crest. Proc Natl Acad Sci USA 107: 2066-2071.

CULBERTSON, M.D., LEWIS, Z.R., and NECHIPORUK, A.V. (2011). Chondrogenic and gliogenic subpopulations of neural crest play distinct roles during the assembly of epibranchial ganglia. PLOS ONE 6: e24443.

D'AMICO-MARTELA, and NODEN DM. (1983). Contributions of placodal and neural crest cells to avian cranial peripheral ganglia. Am J Anat 166: 445-468. 
DONA, E., BARRY, J.D., VALENTIN, G., QUIRIN, C., KHMELINSKII, A., KUNZE, A., DURDU, S., NEWTON, L.R., FERNANDEZ-MINAN, A., HUBER, W., KNOP, M., and GILMOUR, D. (2013). Directional tissue migration through a self-generated chemokine gradient. Nature 503: 285-289.

DUTTA, S., DIETRICH, J.E., ASPÖCK, G., BURDINE, R.D., SCHIER, A., WESTERFIELD, M., and VARGA, Z.M. (2005). Pitx3 defines an equivalence domain for lens and anterior pituitary placode. Development 132: 1579-1590.

FRETER, S., FLEENOR, S.J., FRETER, R., LIU, K.J., and BEGBIE, J. (2013). Cranial neural crest cells form corridors prefiguring sensory neuroblast migration. Development 140: 3595-3600.

FRETER, S., MUTA, Y., MAK, S.S., RINKWITZ, S., and LADHER, R.K. (2008). Progressive restriction of otic fate: the role of FGF and Wnt in resolving inner ear potential. Development 135: 3415-3424.

GRAHAM, A., BLENTIC, A., DUQUE, S., and BEGBIE, J. (2007). Delamination of cells from neurogenic placodes does not involve an epithelial-to-mesenchymal transition. Development 134: 4141-4145.

GROVES, A.K., and BRONNER-FRASER, M. (2000). Competence, specification and commitment in otic placode induction. Development 127: 3489-3499.

HANS, S., and WESTERFIELD, M. (2007). Changes in retinoic acid signaling alter otic patterning. Development 134: 2449-2458.

HARDEN, M.V., PEREIRO, L., RAMIALISON, M., WITTBRODT, J., PRASAD, M.K., MCCALLION, A.S., and WHITLOCK, K.E. (2012). Close association of olfactory placode precursors and cranial neural crest cells does not predestine cell mixing. Dev Dyn 241: 1143-1154.

HUANG, J., RAJAGOPAL, R., LIU, Y., DATTILO, L.K., SHAHAM, O., ASHERY-PADAN, R., and BEEBE, D.C. (2011). The mechanism of lens placode formation: a case of matrix-mediated morphogenesis. Dev Biol 355: 32-42.

KOZLOWSKI, D.J., MURAKAMI, T., HO, R.K., and WEINBERG, E.S. (1997). Regional cell movement and tissue patterning in the zebrafish embryo revealed by fate mapping with caged fluorescein. Biochem Cell Biol 75: 551-562.

KNABE, W., OBERMAYER, B., KUHN, H.J., BRUNNETT, G., and WASHAUSEN, S. (2009). Apoptosis and proliferation in the trigeminal placode. Brain Struct Funct 214: 49-65.

KNAUT, H., BLADER, P., STRÄHLE, U., and SCHIER, A.F. (2005). Assembly of Trigeminal Sensory Ganglia by Chemokine Signaling. Neuron 47: 653-666.

KWAN, K.M., OTSUNA, H., KIDOKORO, H., CARNEY, K.R., SAIJOH, Y., and CHIEN, C.B. (2011). A complex choreography of cell movements shapes the vertebrate eye. Development 139: 359-372.

LADHER, R.K., WRIGHT, T.J., MOON, A.M., MANSOUR, S.L., and SCHOENWOLF, G.C. (2005). FGF8 initiates inner ear induction in chick and mouse. Genes Dev 19: 603-613.

LASSITER, R.N.T., REYNOLDS, S.B., MARIN, K.D., MAYO, T.F., and STARK, M.R. (2009). FGF signaling is essential for ophthalmic trigeminal placode cell delamination and differentiation. Dev Dyn 238: 1073-1082.

LECAUDEY, V., CAKAN-AKDOGAN, G., NORTON, W.H.J., and GILMOUR, D. (2008). Dynamic Fgf signaling couples morphogenesis and migration in the zebrafish lateral line primordium. Development 135: 2695-2705.

LEWELLIS, S.W., NAGELBERG, D., SUBEDI, A., STATON, A., LEBLANC, M., GIRALDEZ, A., and KNAUT, H. (2013). Precise SDF1-mediated cell guidance is achieved through ligand clearance and microRNA-mediated decay. J Cell Biol 200: 337-355.

LLERAS-FORERO, L. and STREIT, A. (2012). Development of the sensory nervous system in the vertebrate head: the importance of being on time. Curr Opin Genet Dev 22: 315-322.

MA, E.Y., and RAIBLE, D.W. (2009). Signaling pathways regulating zebrafish lateral line development. Curr Biol 19: R381-R386.

MAIER, E., HOFSTEN, VON, J., NORD, H., FERNANDES, M., PAEK, H., HEBERT, J.M., and GUNHAGA, L. (2010). Opposing Fgf and Bmp activities regulate the specification of olfactory sensory and respiratory epithelial cell fates. Development 137: 1601-1611.

MARTIN, K., and GROVES, A.K. (2006). Competence of cranial ectoderm to respond to Fgf signaling suggests a two-step model of otic placode induction. Development 133: 877-887.

MCCABE, K.L., SECHRIST, J.W., and BRONNER-FRASER, M. (2009). Birth of ophthalmic trigeminal neurons initiates early in the placodal ectoderm. $J$ Comp Neurol 514: 161-173.
MCCARROLL, M.N., LEWIS, Z.R., CULBERTSON, M.D., MARTIN, B.L., KIMELMAN, D., and NECHIPORUK, A.V. (2012). Graded levels of Pax2a and Pax8 regulate cell differentiation during sensory placode formation. Development 139: 2740-2750.

MIYASAKA, N., KNAUT, H., and YOSHIHARA, Y. (2007). Cxcl12/Cxcr4 chemokine signaling is required for placode assembly and sensory axon pathfinding in the zebrafish olfactory system. Development 134: 2459-2468.

NECHIPORUK, A., LINBO, T., POSS, K.D., and RAIBLE, D.W. (2006). Specification of epibranchial placodes in zebrafish. Development 134: 611-623.

NIKAIDO, M., DOI, K., SHIMIZU, T., HIBI, M., KIKUCHI, Y. and YAMASU, K. (2007) Initial specification of the epibranchial placode in zebrafish embryos depends on the fibroblast growth factor signal. Dev Dyn 236: 564-571.

OHYAMA, T. and GROVES, A.K. (2004). Generation of Pax2-Cre mice by modification of a Pax2 bacterial artificial chromosome. Genesis 38: 195-199.

O'NEILL, P., MAK, S.S., FRITZSCH, B., LADHER, R.K., and BAKER, C.V. (2012) The amniote paratympanic organ develops from a previously undiscovered sensory placode. Nat Commun 3: 1041.

PADANAD, M.S., BHAT, N., GUO, B., and RILEY, B.B. (2012). Conditions that influence the response to Fgf during otic placode induction. Dev Biol 364: 1-10.

PADANAD, M.S., and RILEY, B.B. (2011). Pax2/8 proteins coordinate sequential induction of otic and epibranchial placodes through differential regulation of foxi1, sox3 and fgf24. Dev Biol 351: 90-98.

PIEPER, M., EAGLESON, G.W., WOSNIOK, W., and SCHLOSSER, G. (2011). Origin and segregation of cranial placodes in Xenopus laevis. Dev Biol 360: 257-275.

SAI, X., and LADHER, R.K. (2008). FGF signaling regulates cytoskeletal remodeling during epithelial morphogenesis. Curr Biol 18: 976-981.

SCHLOSSER, G. (2010). Making Senses: Development of Vertebrate Cranial Placodes, 1st ed. Int. Rev. Cell Mol. Biol. 283: 129-234.

SCHLOSSER, G., and AHRENS, K. (2004). Molecular anatomy of placode development in Xenopus laevis. Dev Biol 271: 439-466.

SCHNEIDER-MAUNOURY, S., and PUJADES, C. (2007). Hindbrain signals in otic regionalization: walk on the wild side. Int J Dev Biol 51: 495-506.

SHIAU, C.E., and BRONNER-FRASER, M. (2009). N-cadherin acts in concert with Slit1-Robo2 signaling in regulating aggregation of placode-derived cranial sensory neurons. Development 136: 4155-4164.

SHIAU, C.E., DAS, R.M., and STOREY, K.G. (2011). An effective assay for high cellular resolutiontime-lapse imaging of sensory placode formation. BMC Neuroscience 12: 37.

SHIAU, C.E., LWIGALE, P.Y., DAS, R.M., WILSON, S.A., and BRONNER-FRASER, M. (2008). Robo2-Slit1 dependent cell-cell interactions mediate assembly of the trigeminal ganglion. Nat Neurosci 11: 269-276.

SHIGETANI, Y., HOWARD, S., GUIDATO, S., FURUSHIMA, K., ABE, T., and ITASAKI, N. (2008). Wise promotes coalescence of cells of neural crest and placode origins in the trigeminal region during head development. Dev Bio/319: 346-358.

STARK, M.R., SECHRIST, J., BRONNER-FRASER, M., and MARCELLE, C. (1997) Neural tube-ectoderm interactions are required for trigeminal placode formation. Development 124: 4287-4295.

STREIT, A. (2002). Extensive Cell Movements Accompany Formation of the Otic Placode. Dev Biol 249: 237-254.

STREIT, A. (2008). The cranial sensory nervous system: specification of sensory progenitors and placodes. StemBook [Internet]. Cambridge (MA): Harvard Stem Cell Institute.

SUN, S.K., DEE, C.T., TRIPATHI, V.B., RENGIFO, A., HIRST, C.S., and SCOTTING P.J. (2007). Epibranchial and otic placodes are induced by a common Fgf signal, but their subsequent development is independent. Dev Biol 303: 675-686.

THEVENEAU, E., STEVENTON, B., SCARPA, E., GARCIA, S., TREPAT, X., STREIT, A., and MAYOR, R. (2013). Chase-and-run between adjacent cell populations promotes directional collective migration. Nat Cell Biol 15: 763-772.

TORO, S., and VARGA, Z.M. (2007). Equivalent progenitor cells in the zebrafish anterior preplacodal field give rise to adenohypophysis, lens, and olfactory placodes. Semin Cell Dev Biol 18: 534-542.

VENKITESWARAN, G., LEWELLIS, S.W., WANG, J., REYNOLDS, E., NICHOLSON, C., and KNAUT, H. (2013). Generation and dynamics of an endogenous, self-generated signaling gradient across a migrating tissue. Cell 155: 674-871.

WASHAUSEN, S., and KNABE, W. (2012). Apoptosis contributes to placode morphogenesis in the posterior placodal area of mice. Brain Struct Funct 218: 789-803. 
WASHAUSEN, S., OBERMAYER, B., BRUNNETT, G., KUHN, H.J., and KNABE, W. (2005). Apoptosis and proliferation in developing, mature, and regressing epibranchial placodes. Dev Biol 278: 86-102.

WHITLOCK, K.E., and WESTERFIELD, M. (2000). The olfactory placodes of the zebrafish form by convergence of cellular fields at the edge of the neural plate. Development 127: 3645-3653.
XIONG, F., TENTNER, A.R., HUANG, P., GELAS, A., MOSALIGANTI, K.R., SOUHAIT, L., RANNOU, N., SWINBURNE, I.A., OBHOLZER, N.D., COWGILL, P.D., SCHIER, A.F., and MEGASON, S.G. (2013). Specified neural progenitors sort to form sharp domains after noisy Shh signaling. Cell 153: 550-561.

XU, H., DUDE, C.M., and BAKER, C.V. (2008). Fine-grained fate maps for the ophthalmic and maxillomandibular trigeminal placodes in the chick embryo. Dev Biol 317: 174-186. 


\section{Further Related Reading, published previously in the Int. J. Dev. Biol.}

Clonal analyses in the anterior pre-placodal region: implications for the early lineage bias of placodal progenitors Sujata Bhattacharyya and Marianne E. Bronner Int J Dev Biol. (2013) 57:753-757

The surface ectoderm of the chick embryo exhibits dynamic variation in its response to neurogenic signals

Vineeta-Bhasker Tripathi, Yasuo Ishii, Muhammad M. Abu-Elmagd and Paul J. Scotting Int. J. Dev. Biol. (2009) 53: 1023-1033

http://dx.doi.org/10.1387/ijdb.082780vt

The preplacodal region: an ectodermal domain with multipotential progenitors that contribute to sense organs and cranial sensory ganglia Andrea Streit

Int J Dev Biol (2007) 51:447-461

The molecular biology of ear development - "Twenty years are nothing"

Fernando Giraldez and Bernd Fritzsch

Int. J. Dev. Biol. (2007) 51: 429-438

http://dx.doi.org/10.1387/ijdb.072390fg

Hindbrain signals in otic regionalization: walk on the wild side

Sylvie Schneider-Maunoury and Cristina Pujades

Int. J. Dev. Biol. (2007) 51: 495-506

http://dx.doi.org/10.1387/ijdb.072345ss

5 yr ISI Impact Factor $(2011)=2.959$
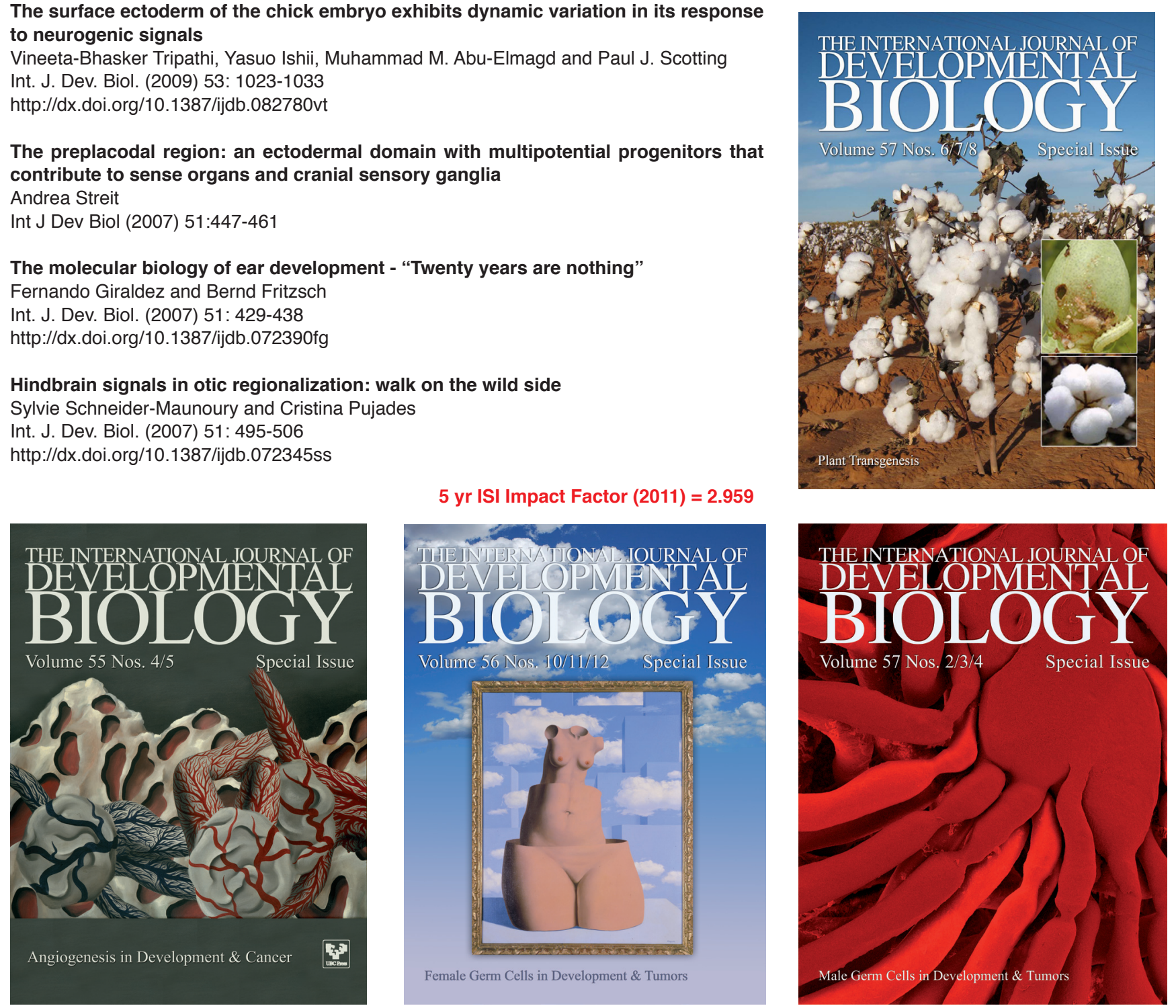